\title{
Phytopathogenic organisms and mycotoxigenic fungi: why do we control one and neglect the other? a biological control perspective in Malaysia
}

\begin{abstract}
In this review, we present the current information on development and applications of biological control against phytopathogenic organisms as well as mycotoxigenic fungi in Malaysia as part of the integrated pest management (IPM) programs in a collective effort to achieve food security. Although the biological control of phytopathogenic organisms of economically important crops is well established and widely practiced in Malaysia with considerable success, the same cannot be said for mycotoxigenic fungi. This is surprising because the year round hot and humid Malaysian tropical climate is very conducive for the colonization of mycotoxigenic fungi and the potential contamination with mycotoxins. This suggests that less focus has been made on the control of mycotoxigenic species in the genera Aspergillus, Fusarium, and Penicillium in Malaysia, despite the food security and health implications of exposure to the mycotoxins produced by these species. At present, there is limited research in Malaysia related to biological control of the key mycotoxins, especially aflatoxins, Fusarium-related mycotoxins, and ochratoxin A, in key food and feed chains. The expected threats of climate change, its impacts on both plant physiology and the proliferation of mycotoxigenic fungi, and the contamination of food and feed commodities with mycotoxins, including the discovery of masked mycotoxins, will pose significant new global challenges that will impact on mycotoxin management strategies in food and feed crops worldwide. Future research, especially in Malaysia, should urgently focus on these challenges to develop IPM strategies that include biological control for minimizing mycotoxins in economically important food and feed chains for the benefit of ensuring food safety and food security under climate change scenarios.
\end{abstract}

